\title{
Compare the Cognitive Abilities and Subjective Well-Being of Elderly Living With Families and Old Age Homes
}

\author{
Jyotsana Maurya $^{1 *}$, Dr. Kiran U.V. ${ }^{2}$
}

\section{ABSTRACT}

Aging is not a disease, but the final stage of normal life. Cognitive abilities mean conscious mental activity as thinking, remembering, learning or using language. Considerable work has been done to understand the age-related decline in cognitive abilities. Subjective well-being is defined as people's evaluation of their own lives. Such evaluations can be both cognitive judgements, such as life satisfaction, and emotional responses to events, such as feeling positive emotions. The present study focuses on Compare the cognitive abilities and subjective wellbeing of elderly living with families and old age homes. The study was carried out in lucknow. Random sampling technique was fallowed in the present study. The sample for this study comprised of 120 elderly individuals (60 females and 60 males respectively) from urban and semi-urban areas. Cognitive abilities scale: The cognitive abilities scale of elderly was assessed using cognitive abilities scale by Dr. Avishai Antonovsky (1987). Subjective Well-Being scale: The perception of Subjective well-being among elderly was measured using Subjective wellbeing scale developed by Prof. William Pavot. et al (1997)., The sample of this study were personally and individually contacted and data was obtained through face-to face interview. The positive clearly indicates that more cognitive abilities better will be the subjective well-being among elderly living at various places.

Keywords: Cognitive Abilities, Subjective Well-Being, Elderly.

Aging is not a disease, but the final stage of normal life. Aging population is one of the most significant characteristics of the $21^{\text {st }}$ century and is one of the most pressing issues faced by all regions of the world. The issue of aging of population is of great concern particularly for Japan and India. Addressing the issues of elderly in their socioeconomic arena and factors related to their satisfaction with life has significant research implications. Japan provides an interesting

\footnotetext{
${ }^{1}$ Research Scholar, Department Of Human Development And Family Studies, School For Home Sciences, Babashaheb Bhimrao Ambedker University, Lucknow, India

${ }^{2}$ Assistant Professor, Department Of Human Development And Family Studies, School For Home Sciences, Babashaheb Bhimrao Ambedker University, Lucknow, India

*Responding Author

(C) 2016 Maurya J, Kiran UV; licensee IJIP. This is an Open Access Research distributed under the terms of the Creative Commons Attribution License (http://creativecommons.org/licenses/by/2.0), which permits unrestricted use, distribution, and reproduction in any Medium, provided the original work is properly cited.
} 


\section{Compare the Cognitive Abilities and Subjective Well-Being of Elderly Living With Families and Old Age Homes}

setting for the study as it has the highest longevity in the world. By, 2010, there will be 29.29 million elderly (65 years and older) constituting $23.1 \%$ of the total population in Japan marking record heights both in number and percentage .Japanese have the highest life expectancy in the World (86.39 years for women and 79.64 years for men in 2010) (Ministry of Internal Affairs and Communications 2011).

Aging of population is an end product of demographic transition. The number of elderly people in developing countries is almost 3-4 times of that of developed countries. Increased human life span as witnessed in the preceding decades has not been accompanied by good quality of life for majority of older Indians. World population of 6.1 billion at the dawn of $21^{\text {st }}$ century is likely to become 9.3 billion in 2050 (UN 2004).

Global aged population is going to increase from 595 million to 2 billion-a fourfold rise-by 2050. In terms of proportion rise was $10 \%$ in $2000,15 \%$ by 2025, predicted to rise to $21.6 \%$ by 2050 . Nearly 90 per cent of the total workforce in India is employed in the unorganised sector. Consequently, retirement from gainful employment precludes financial security like pension and other post-retirement benefits. It is estimated that one-third of the elderly population live below the poverty line. As majority works in unorganized sector the dependence rate of the elderly is also so high.

Cognitive functioning and Subjective well-being might be those two main aspects that can help in creating policies that may lead people to a contented and independent way of living. Good strategy in this regard would be to start from middle age onward for preventing the causes of complications in later years of life instead of just providing support to elderly people in their in capability. It might be more beneficial if we able to find such factors which are not only positively associated with both cognition and subjective well-being but also contribute in effecting their relationship positively. Cognitive abilities have a very important role in daily life of any individual as whatever one does depends on the level of his understanding about the task and its context. Nothing is beyond the domain of cognitive abilities whether it is related to selfcare like bathing or interacting with people or solving a complex problem at work etc. The term "Cognitive” according to the Webster's Dictionary means conscious mental activity as thinking, remembering, learning or using language.

Considerable work has been done to understand the age-related decline in cognitive abilities. Human cognitive abilities are divided into two aspects by researchers, first, crystallized intelligence, includes verbal ability, numerical skills and general knowledge which shows little decline due to ageing. Second, the fluid intelligence, which includes aspects of memory, executive functions, processing speed and reasoning, start declining from middle age onwards oreven earlier than this. These fluid intelligent aspects of cognition are those which help in 


\section{Compare the Cognitive Abilities and Subjective Well-Being of Elderly Living With Families and Old Age Homes}

performing daily activities and ensure an autonomous, functional life. Moreover, decline in anyone domain of fluid intelligence effects the others domains so they start declining also.

Cognitive abilities change over the lifespan and in older age differences between and within individuals becomes more pronounced. Highly over learned functions as assessed by using implicit or procedural memory tasks (for instance, brushing your teeth, playing the piano or riding a bicycle), and crystallized intelligence (assessed by using semantic knowledge tasks, such as those testing vocabulary, knowledge, culture, etc.) appear to be stable with age. However, other cognitive abilities show decrements with age, for example, those assessed using tests requiring processing speed. This is partly because of a general slowing of sensor motor speed.

This slowing is additionally pronounced in more complex choice reaction time tests. This in turn is probably related to less efficient or slower executive processes, which can be seen in tests assessing cognitive flexibility, and tasks which require working memory manipulation. Test performance on these tasks declines, albeit at different ages of onset and with different rates of decline. For example, the Seattle Longitudinal Study, which followed participants over a period of 35 years, found that perceptual speed already started to decline in the mid-50s, while verbal memory performance did not decline until the late 70s. Similar differential declines, with performance on perceptual speed and fluid intelligence tasks decreasing much earlier than performance on verbal memory or crystallized intelligence tasks, have been found in a large number of studies using different tests to assess cognitive abilities (summarized). Maintaining cognitive ability will also depend on mood, morbidity and health status of participants, which can all accelerate decline with age. For instance, thyroid status, which can occur in $6 \%$ of elderly who view themselves as healthy, can significantly affect cognitive function and decline. Depression, which can have a profound effect on cognitive ability, is quite common in those over 65 years of age and is estimated to affect around 1 in 5 older individuals. Age-related pathological cognitive conditions, such as dementia, further accelerate the rate of decline of cognitive abilities, but again this shows a differential pattern, with even higher variability than in the normal aging process. In addition, dementia in its later stages also affects those cognitive abilities that are stable in normal aging (for instance, semantic memory and knowledge). Finding older people without any morbidity that can affect cognitive ability is difficult, although with improved health care many elderly are living longer healthier. Brar et al. (2013)in their study on "Mental Health of Elderly as Related to Their Well-being and Self Esteem" The present investigation was conducted to gain an insight into the Mental Health of Elderly as Related to their Well Being and Self Esteem from middle income group belonging to the urban areas of Chandigarh. The study was conducted on 100 elderly out of whom 50 were females and 50 males belonging to the age group of 60-65 years. Standardized tools namely Friedman-wellbeing, Self-esteem inventory and Mental Health Inventory were used. The results showed that correlation between some of the variables of Wellbeing, Self-esteem and mental health were 


\section{Compare the Cognitive Abilities and Subjective Well-Being of Elderly Living With Families and Old Age Homes}

significant. Multiple regression analysis showed that some of the variables of well-being and Self-esteem predicted mental health among elderly.

“Subjective well-being is defined as people's evaluation of their own lives. Such evaluations can be both cognitive judgements, such as life satisfaction, and emotional responses to events, such as feeling positive emotions. Subjective well-being is thus an umbrella term that refers to several separable components: life satisfaction and satisfaction with life domains such as marriage, work, income, housing and leisure; feeling positive affect (pleasant emotions and moods) most of the time; experiencing infrequent feelings of negative affect (such as depression, stress, and anger); and judging one’s life to be fulfilling and meaningful.” (Diener\& Biswas-Diener, 2003) Overall, subjective well-being is a broad construct that involves the combination of three particular factors: frequent and intense positive affective states by experiencing positive emotions, the relative absence of anxiety and depression with a low level of negative moods, and high global life satisfaction (Diener et al., 2002).

Global population aging is unprecedented in history and will have far-reaching consequences for the social, economic, and political landscapes of countries over the next several decades. Each country's response to population aging depends, in part, on how well the older population fares now and how well it is likely to fare in the future. But the range of economic, social and health factors that affect elderly well-being makes planning and responding to population aging difficult. Predicting the effect of national policies and programs on elderly well-being is also a challenge. An index of elderly well-being that is comparably measured across several countries can help with both these issues. This fact sheet introduces such an index-the SCL/PRB Index of Well-Being in Older Populations-developed by the Stanford Center on Longevity (SCL) and the Population Reference Bureau (PRB). The SCL/PRB Index summarizes the status of the elderly across multiple dimensions and allows for the possibility of comparing the well-being of older populations across countries with key differences in policies or programs targeting older people.

The SCL/PRB Index aids in assessing the overall well-being of older population groups of one country relative to others and in ascertaining which factors contribute to a country's relative standing. The index summarizes 12 key indicators of well-being in old age across four domains — material, physical, social, and emotional wellbeing domains:

Material well-being captures the extent to which the older population may be able to meet their needs for basic goods such as food and shelter. Physical and cognitive well-being assesses the ability to perform basic activities of daily living and to live independently, with life expectancy as the most fundamental outcome at the population level. Social engagement measures involvement with family members, peers, community members, and local institutions to gauge 


\section{Compare the Cognitive Abilities and Subjective Well-Being of Elderly Living With Families and Old Age Homes}

the levels of social participation and the availability of social support. In addition to being associated with better health, these contribute to life satisfaction. Emotional well-being measures mental and psychological outlook. It is closely tied to both physical health and social support.

Though well-being varies over the life span, it often does so in a manner that commonsense would not predict. Aswe indicated, findings show elderly persons to be generally more satisfied with their lives than other age groups, to worry less, except in regard to health, and to evidence less negative affect and depression. The stereotypes of older age as a period of decline in the quality of life do not hold up. In speaking of elderly persons, as with any large social category, we must interject a note of caution, for they are a heterogeneous group. AsBlau insightfully points out, structural factors such as education, ethnicity, employment, and marital status mediate the effects of age upon physical and mental health and self-conception. In order to develop a more precise understanding of well-being among elderly persons, we need to focus upon particular aged populations and the psychosocial factors that influence their well-being. Meddin et al. (1988)has conducted a study on "Subjective Well-being among the Rural Elderly" The purpose of this study was to investigate the relationship between psychosocial factors and subjective well-being among rural elderly persons. Abroad range of psychosocial factors (such as mastery, social support, and perceived health) and measures of well-being (such as positive and negative affect and life satisfaction) were employed in bivariate correlation, canonical correlation, and multiple regression analysis. The findings show significant relationships between subjective well-being measures and psychosocial factors that are concomitant with other populations, both elderly and general. Rathore (1992) investigated the subjective well-being of middle-aged and older men, using measures of quality of life, well-being, self-esteem, positive negative affect. Older men were found to have poorer quality of life, poor subjective well-being and self-esteem compared to middle aged men. Wolinsky et al.(1985 ) has conducted a study on "Correlates of change in subjective wellbeing among the elderly" This study examines two related issues concerning the subjective wellbeing Of elderly adults: change over time and correlates of that change. Data come from a three wave panel study of 401 elderly residents in St. Louis. Residualized change score regression analyses indicate: there is change in subjective wellbeing over 4-5 months and over 12 months; the $4-5$ month and 12 month changes are remarkably similar; the effect of subjective wellbeing over time indicates regression to the mean; and only socioeconomic status is a significant predictor of change in subjective wellbeing (net of the effects of subjective wellbeing itself). The implications of these results for our understanding of subjective wellbeing in the elderly are discussed, as are the policy implications of the positive effect of socioeconomic status on changes in the subjective wellbeing of the elderly.

\section{Objective}

- To compare the cognitive abilities and subjective well-being of elderly living with families and old age homes. 


\section{Compare the Cognitive Abilities and Subjective Well-Being of Elderly Living With Families and \\ Old Age Homes}

\section{METHODOLOGY}

The sample for this study comprised of 120 elderly individuals (60 females and 60 males respectively) from urban and semi urban areas of Lucknow district.

\section{Sampling design}

Lucknow city was purposively selected to conduct the study.

Random sampling technique was followed in the present study.

\section{Tools and techniques}

To carry out the present study, the following tools were used to measure various parameters.

\section{Cognitive abilities scale:}

The cognitive abilities scale of elderly was assessed using cognitive abilities scale by Dr. Avishai Antonovsky (1987).

\section{Subjective Well-Being scale:}

The perception of Subjective well-being among elderly was measured using Subjective wellbeing scale developed by Prof. William Pavot. et al (1997).

\section{Procedure}

The samples of this study were personally and individually contacted and data was obtained through face-to face interview. The duration of data collection were spread over a period of three months (60 days). The obtained responses were scored and statistically analyzed.

\section{Data processing}

Coding-_Coding refers to the process of assigning, numbers other symbols to answers. So that workers on them be part into a limited number of categories or classes appropriate to the research problem under consideration.

Scoring- The scoring of various dependent and independent variables include in the questionnaire was done as mentioned below

\section{Data Analysis:}

Data collected was analysed statistically with the help of various statistical techniques using PAS software. 
RESULT

Table 1, Assessment of cognitive abilities among elderly living with families \& old age homes of various age group.

\begin{tabular}{|c|c|c|c|c|c|c|c|c|}
\hline \multirow[t]{2}{*}{ Statement } & \multicolumn{2}{|l|}{ Old age } & \multirow[t]{2}{*}{ t } & \multirow[t]{2}{*}{$\mathbf{p}$} & \multicolumn{2}{|l|}{ With family } & \multirow[t]{2}{*}{$\mathbf{t}$} & \multirow[t]{2}{*}{$\mathbf{P}$} \\
\hline & $60-70$ & $71-80$ & & & $60-70$ & $71-80$ & & \\
\hline $\begin{array}{l}\text { Cognitive } \\
\text { abilities }\end{array}$ & $100.9 \pm 12.001$ & $112.14 \pm 14.250$ & 2.823 & .098 & $102.54 \pm 17.200$ & $104.36 \pm 9.927$ & 15.206 & .000 \\
\hline
\end{tabular}

From the above table no.1 it was shown that there exists significant difference of elderly according to various age groups in old age home and living with family. Data shows that p value in old age home was more than 0.05 in parameters like cognitive abilities among elderly of various age groups in old age home. Data shows that $\mathrm{p}$ value of living within family was less than .000 in parameters like cognitive abilities among elderly of various age groups in living wit in family. The hypothesis was highly significant. The null hypothesis was rejected.

Table. 2-Assessment of subjective well-being among elderly living with family and old age home on various age groups.

\begin{tabular}{|l|l|l|l|l|l|l|l|l|}
\hline Statement & \multicolumn{2}{l|}{ Old age } & t & p & \multicolumn{2}{l|}{ With family } & t & P \\
\cline { 2 - 6 } & $\mathbf{6 0 - 7 0}$ & $\mathbf{7 1 - 8 0}$ & & & $\mathbf{6 0 - 7 0}$ & $\mathbf{7 1 - 8 0}$ & & \\
\hline $\begin{array}{l}\text { Subjective } \\
\text { well-being }\end{array}$ & $3.16 \pm 1.620$ & $4.18 \pm 1.736$ & 1.281 & .262 & $3.24 \pm 1.876$ & $3.93 \pm 0.997$ & 15.846 & .000 \\
\hline
\end{tabular}

From the above table no.2 it was shown that there exists significant difference of elderly of various age groups in old age home and living with family. Data shows that $\mathrm{p}$ value in old age home was more than 0.05 in parameters like subjective well-being among elderly of various age groups in old age home. Data shows that $\mathrm{p}$ value of living within family was less than 0.05 in parameters like subjective well-being among elderly of various age groups in living with in family. The hypothesis was highly significant. The null hypothesis was accepted.

Relationship with cognitive abilities and subjective well-being among elderly

\begin{tabular}{|l|l|l|}
\hline Statement & Cognitive scale & Subjective scale \\
\hline Cognitive scale & 1 & \\
\hline Subjective scale & $0.403^{* *}$ & 1 \\
& 0.000 & \\
\hline
\end{tabular}

It is also evident that the cognitive abilities and subjective well-being are highly correlated. The positive clearly indicates that more cognitive abilities better will be the subjective well-being among elderly living at various places.

(c) The International Journal of Indian Psychology, ISSN 2348-5396 (e)| ISSN: 2349-3429 (p) | 157 


\section{Compare the Cognitive Abilities and Subjective Well-Being of Elderly Living With Families and Old Age Homes}

\section{RESULT AND DISCUSSION}

Result was shown that there exists significant difference of elderly according to various age groups in old age home and living with family. Data shows that the cognitive abilities among elderly living with families were found to be higher in comparison to the elderly living in old age homes. This may be due to the reason that elderly living in old age homes are stressed as they have to live leaving their families. It may be noted that the subjective wellbeing of the elderly living with families was found to be better in comparison to the elderly living in old age homes. The purpose of this study was to examine the pattern of cognitive function and subjective wellbeing among the different age groups of elderly people and the compare these important aspects of successful ageing. Our hypotheses were that higher cognitive function results in better subjective well-being in ageing. The results of this study support one of the hypotheses that higher level of cognitive abilities is associated with good subjective well-being. According to their findings higher cognitive abilities was also one of the factors which were positively related to subjective quality of live.

\section{CONCLUSION}

The challenge we are facing currently is not regarding the increase in number of years in life of people but how can we make them able to live a healthy and independent life. It might not be realistic if we think healthy and independent means a disease free life in older age. So focus is shifted towards the cognitive abilities and subjective well-being aspects of success full ageing. Moreover as it is not possible to ignore the effects of ageing itself on cognitive abilities so it might be more beneficial if we start maintaining the cognitive abilities reserves from the mid-life age so that not only process of natural decline in cognition can be delayed but also give boost to subjective well-being. In this study the pattern of cognition and subjective well-being were emphasized and related. This effort was to identify such factors which may shed some light on the nature of relationship existing between cognitive abilities and subjective well-being.

\section{Acknowledgments}

The author appreciates all those who participated in the study and helped to facilitate the research process.

\section{Conflict of Interests}

The author declared no conflict of interests.

\section{REFFERENCES}

Alesina, Alberto, Edward Glaeser, and Bruce Sacerdote. 2005. "Work and Leisure in the U.S. and Europe: Why so Different?” NBER Working Paper No. 11278.

Burse W. Ewald Handbook of Geriatric Psychiatry. New York :Van Nastran Reinhold co publishers, 1980: 22243 


\section{Compare the Cognitive Abilities and Subjective Well-Being of Elderly Living With Families and Old Age Homes}

Casel CK. Use it or lose it: Activity may be the best treatment for Aging. Journal of the American Medical Association. 2002;288(18):2333-2334.

Christensen K, Doblhammer G, Rau R, Vaupel JW. Ageing populations: the challenges ahead. Lancet. Elsevier Ltd; 2009 Oct 3; 374(9696):1196-208.

Deary IJ, Corley J, Gow AJ, Harris SE, Houlihan LM, Marioni RE, et al. (2009 Jan)Ageassociated cognitive decline. British medical bulletin; 92:135-52.

Di Tella, Rafael, Robert J. McCulloch, and Andrew J. Oswald. 2001. "Preferences Over Inflation and Unemployment.” American Economic Review 91(1): 335-341.

Easterlin R, Angelescu L. (2007) Modern Economic Growth and Quality of Life: Cross Sectional and Time Series Evidence. University of Southern California.; (2755).

Easterlin, Richard. 1974. "Does Economic Growth Improve the Human Lot? Some Empirical Evidence.” In Paul David and Melvin Reder, eds, Nations and Households in Economic Growth: Essays in Honor of Moses Abromovitz. London: Academic Press, 98-125.

Fillit H, Butler R, O’Connell A, Albert M, Birren J, Contman C, et al. Achieving and maintaining cognitive vitality with aging. Mayo Clinic Proceedings. 2002;77(7):681-696.

Gruber, Jonathan and Sendhil Mullainathan. 2002. "Do Cigarette Taxes Make Smokers Happier?" NBER Working Paper No. 8872.

Helliwell, John F. 2006. "Well-Being, Social Capital and Public Policy: What's New?" Economic Journal 116(510): C34-C45.

http://www.un.org/esa/population/publications/WPP2004/2004Highlights_finalrevised.pdf

Huppert F. (2009 Jul) Psychological Well-being: Evidence Regarding its Causes and Consequences. Applied Psychology: Health and Well-Being 2009 Jul ; 1(2):137-64.

Kiran, U.V. \& Maurya J. (2015).E-culture and its impact on elderly's loneliness and life satisfaction, 2,3.4.

Kozma, A., and Stones, M.J., McNeil, J.K. (1991)., Psychological well-being in later life., Toronto, Canada: Butterworths.

Lunenfeld B. The ageing male: demographics and challenges. World journal of urology [Internet]. 2002 [cited 2012 Aug 24]; Available from:

McGillivray M, Clarke M, ebrary, Inc. Understanding human well-being [Internet]. 2006 [cited 2011 December 31]. Available from: http://archive.unu.edu/unupress/samplechapters/1130-

Park K Text book of Preventive and Social Medicine. Jabalpur :M/s Banarsidas Bhanot, 2002: 40810. Patricia A Potter. Fundamentals of nursing. Missoure : Mosby's publication, 2001 $: 24445$

Schaie K, Willis S, Caskie G. The Seattle longitudinal study: Relationship between personality and cognition. Neuropsychology of Cognition. 2004;11:304-324.

Sell and Nagpal., (1992) Assessment of subjective well-being., World Health Organisation., Regional Office for South - East Asia, New Delhi. 


\section{Compare the Cognitive Abilities and Subjective Well-Being of Elderly Living With Families and Old Age Homes}

Singh-Manoux A, Kivimäki M.( 2010 Dec) The importance of cognitive aging for understanding dementia. Age (Dordrecht, Netherlands). 32(4):509-12.

Teri L, McCurry SM, Logsdon RG. (1997 Oct)Memory, thinking, and aging. What we know about what we know. The Western journal of medicine.; 167(4):269-75.

United Nations. World population prospects. The 2004 revision. (Highlights) [Internet]. 2005 [cited 2011 Dec 24]. Available from:

Waxman HM, Carner EA. Depressive symptoms and health service utilization among the community elderly. Journal of the American geriatric society 1982 Oct; 17 (4) : 1214

Wolfers, Justin. 2003. "Is Business Cycle Volatility Costly? Evidence from Surveys of Subjective Well-being."International Finance 6(1): 1-23.

Wolfers, Justin. 2003. "Is Business Cycle Volatility Costly? Evidence from Surveys of Subjective Well-being."International Finance 6(1): 1-23.

How to cite this article: Maurya J, Kiran UV (2016), Compare the Cognitive Abilities and Subjective Well-Being of Elderly Living With Families and Old Age Homes, International Journal of Indian Psychology, Volume 4, Issue 1, No. 80, ISSN:2348-5396 (e), ISSN:2349-3429 (p), DIP:18.01.116/20160401, ISBN:978-1-365-57867-0 